\title{
Thermodynamic Behaviors of Macroscopic Liquid Droplets Evaporation from Heated Substrates
}

\author{
Xue Chen ${ }^{1} \cdot$ Zhi-Qiang Zhu ${ }^{1}$ - Qiu-Sheng Liu ${ }^{1} \cdot$ Xu-Wen Wang ${ }^{1}$
}

Received: 14 November 2014 / Accepted: 24 March 2015 / Published online: 9 April 2015

(C) Springer Science+Business Media Dordrecht 2015

\begin{abstract}
Evaporation of a macroscopic-scale sessile droplet on different hot isothermal substrates has been experimentally investigated, for the framework of planning space experiments onboard Chinese recoverable satellite to explore the interface effect, heat and mass transfer during the phase transition process. Undoubtedly, the evaporation phenomenon of a sessile drop on heated substrates is a complex problem which involves the behavior of triple line, heat transfer with thermal conduction and convection, mass transfer into the vapor phase. Therefore, preparations from scientific view have been carried out to validate setup of the space experiment modes. Based on the experiments performed in the terrestrial gravity, we found that the evolution of a water droplet could be separated into three stages, began with the constant contact area, then switched to the depin stage and ended up with the flushing stage. The average evaporation rate was measured and the thermal effects of different substrates were studied. Results revealed a linear variation of contact diameter with its average evaporation rate, which has the similar tendency with small drops. The varieties of the heat flux density during evaporating showed that droplet absorbed energy from the heated substrate, then with the help of the internal flow of thermocaplliry and buoyant convection, heat was transported to the liquid-vapor interface providing the energy for evaporation.
\end{abstract}

Qiu-Sheng Liu

liu@imech.ac.cn

1 Key Laboratory of Microgravity (National Microgravity

Laboratory), Institute of Mechanics,

Chinese Academy of Sciences,

Beijing, 100190, China
Keywords Sessile drop evaporation - Thermocaplliry convection - Buoyant convection · Heat and mass transfer

\section{Introduction}

Liquid evaporation is a common phenomenon in nature and also plays an important role in industry due to the high efficiency on heat transfer. In recent years, the evaporation process has been applied to many applications such as spray drying, combustion of fuel, DNA mapping in biochemical areas, microfluidics technique of printing and so on (Erbil 2012; Tadmor and Bahadur 2009; Dittrich and Manz 2006; Jing and Reed 1998; Gogos and Sadhal 1986). However, the mechanism of evaporation process is a complicated problem due to the coupling of the movement of triple line (solid, gas and liquid), heat conduction, thermocapillary and buoyant convection, and diffusion into the vapor phase. Therefore, the investigation of the droplet evaporation has attracted more and more interests.

In the past decades, Picknett and Bexon (1977) was the pioneer in studying the evaporating droplet both experimentally and theoretically. They found out that the presence of two distinguished modes of evaporation: constant contact area and constant contact angle. Bourges-Monnier and Shanahan (1995) and Deegan et al. (2000) performed some similar experiments with different liquids on different substrates focusing on the constant contact area mode. $\mathrm{Hu}$ and Larson (2002) and Hu and Larson (2005) used Finite Element Modele to solve the diffusion-driven evaporation problem and developed a mathematical model to represent droplet evaporation taking Marangoni effect into consideration. In numerical computation aspects, Ruiz and Black (2002) simulated small water droplet regarding internal fluid motion, the internal flow provided vastly different 
temperature distributions in the drop was reported. Saada and Tadrist (2013) modeling the overall evaporation process of pinned and de-pinned modes, in this case both decrease in thermal conductivity of substrate and increase in its thickness had a cooling effect on the drop. Various experimental studies like Sobac and Brutin (2012) and Dunn et al. (2009) had illustrated that the evaporation process was a combination of the dynamics of contact line, fluid dynamics and the thermal properties of the substrate.

Most of the former work assumed that the drop was small enough that the diameter was less than the capillary length $\ell=\sqrt{\sigma / \rho g}$ (equal to $2.72 \mathrm{~mm}$ for water and $1.69 \mathrm{~mm}$ for ethanol), where $\sigma$ is the liquid-gas surface tension, $\rho$ is density and $\mathrm{g}$ is the gravitational acceleration. However, for space experiments, we generally created millimeter-size drops in order to meet the demands of observation and measurement. That means the diameter was larger than the capillary length, few investigations were performed before. The behavior of these droplets remains poorly understood, as demonstrated by Zhu et al. (2012) that buoyancy convection makes a significant contribution on droplet evaporation that confused the problem. By comparison with space experiments, the quantitative analysis of the contribution of different convection could be carried out. In addition, to verify the feasibility of drops evaporation in microgravity and obtain effective techniques for the forthcoming space experiments, we needed to conduct some advanced researches in normal gravity.

In present paper, experimental investigation was carried out to study the evaporation process of macroscopic-scale droplet. In what follows, we would first provide a theoretical analysis of a macroscopic-scale droplet evaporation. Specified description of the experimental apparatus and the measurement techniques were studied in Section "Experimental Setup". In Section "Results and Discussion", we proposed some experiments to find out the universal rules of heat and mass transfer of macroscopic-scale droplet evaporation on different hot isothermal substrates and compared them with small drop. The results about the evolution of the drop shape, variation of the evaporation rate and heat flux density

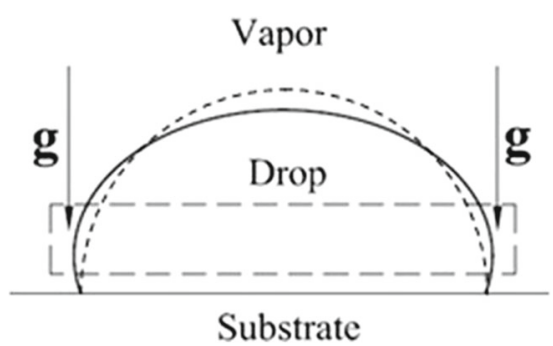

Fig. 1 Schematic of gravitational force influence on the shape of a sessile drop

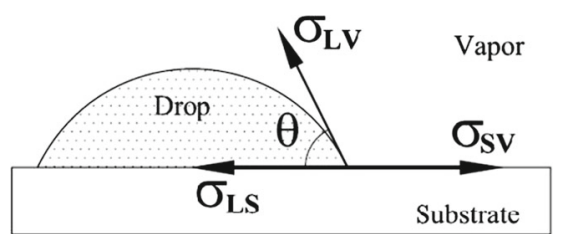

Fig. 2 The resolution of forces at the contact line

were presented and discussed as follows. Finally, comments and conclusions were stated in Section "Conclusion".

\section{Theoretical Analysis}

As shown by previous works, the small droplet adopted a spherical cap shape of which the contact diameter was less than capillary length so that the gravitational force was negligible. However, for macroscopic-scale droplet, the contour mainly balanced by two forces: the surface tension, which had a tendency of minimizing the area of the surface and the gravitational force which tends to flatten the drop. The schematic diagram of gravitational force influence on the shape of a sessile drop was shown in Fig. 1.

The expression for the droplet shape, relating the equilibrium contact angle $\theta$ to the surface tensions acting on the system, was put forward by Young (1805), called Young's equation (1) depicted in Fig. 2, where $\sigma_{L V}, \sigma_{S V}, \sigma_{L S}$ are respectively the surface tension of liquid-vapor, surface energy of solid-vapor and interfacial energy of liquid-solid.

$\sigma_{L V} \cos \theta=\sigma_{S V}-\sigma_{L S}$

Two forms of convection pattern would be mentioned below. The mechanism was plotted in Fig. 3. One could be named after buoyant convection, interpreted as the variation of density gradient under gravity environment. At first, there exists a low-temperature area at the apex of the drop when the substrate was heated, the hot water in the middle flowed upward due to the low density and the cold water downward to replenish the liquid outside. Thus the internal flow along the interface was from the top to the bottom. A non-dimensional Rayleigh number could characterize the instability.

$R_{a}=\frac{\beta g \Delta T h^{3}}{\nu k}$

where $\beta$ is the coefficient of liquid thermal expansion, $\Delta T$ is the temperature difference between the substrate plane

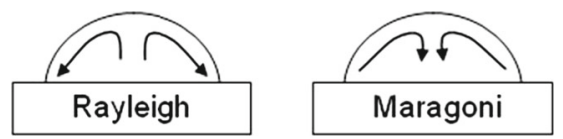

Fig. 3 The mechanism of Rayleigh and Marangoni convection 
Fig. 4 Schematic setup of the experimental apparatus

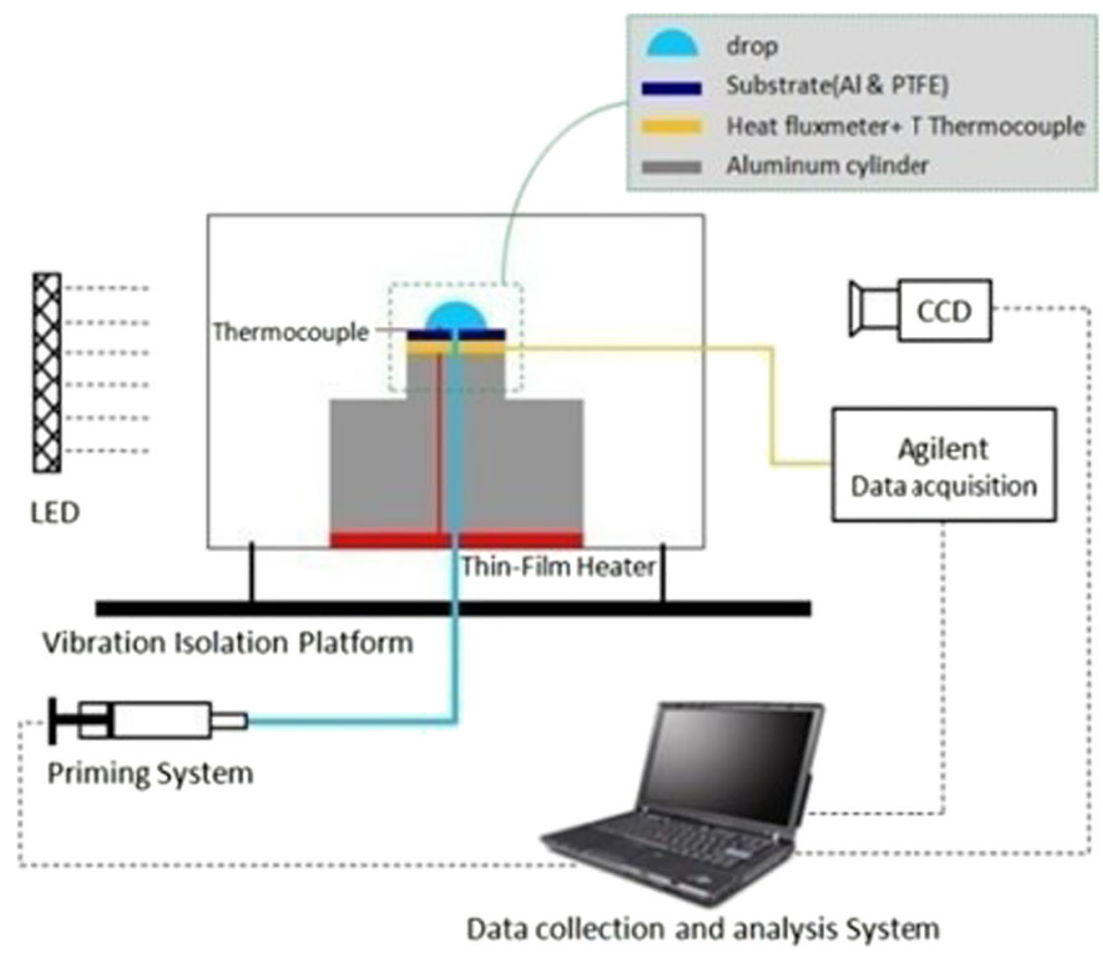

and the apex of the drop, $h$ is the height of the droplet, $v, k$ stand for kinematic viscosity and thermal diffusion coefficient.

Another flow is thermocapillary convection driven by surface tension. We assumed that the temperature along the interface was not isothermal and the liquid was hotter in the region of the triple line than that at the top of the drop, the delay of the thermal conduction must be the reason. Then hot water with low surface tension property would be dragged by the cold water to the top then downward in the middle as supplement. These instability could be reflected by Marangoni number.

$M_{a}=\frac{\sigma_{T} \Delta T h}{\mu k}$

where $\sigma_{T}$ is the temperature coefficient of surface tension, $\mu$ is the dynamic viscosity.

We can see from the above expressions that these two forms of convection would be enhanced by temperature difference, especially for macroscopic-scale liquid droplets, and then the evaporation rate would be accelerated.
For reference, Picknett and Bexon (1977) pointed out that for any shapes of the droplet the rate of mass loss was given by:

$-\frac{d m}{d t}=\pi D R \Delta c_{v} f(\theta)$

According to Picknett theory, when angles were in the range of $0.175<\theta<\pi$ radians, $f(\theta)$ could defined by:

$$
\begin{aligned}
f(\theta)=\frac{1}{2}(0.00008957 & +0.663 \theta+0.116 \theta^{2} \\
& \left.-0.08878 \theta^{3}+0.01033 \theta^{4}\right)
\end{aligned}
$$

Where $t$ is the time, $D$ is the coefficient of vapor diffusion into air, $R$ is the contact radius, $\Delta c_{v}$ is the vapor concentration difference between the interface and $f(\theta)$ is a function of contact angle involving the droplet shape.

These formulas could help us to calculate the evaporation rate for simple diffusion condition. But at hot isothermal substrate, the model mentioned above should be extended to contain the substrate temperature $T_{s}$ and the humidity of the ambient $H . \Delta c_{v}=c_{s}-c_{\infty}$, which is assumed to be saturated at the substrate temperature $c_{s}$ and considered the ambient temperature for $c_{\infty}$. Diffusion coefficient $D\left(T_{S}\right)$

Table 1 Thermophysical properties of fluids at $T_{a}=20{ }^{\circ} \mathrm{C}$ and $P=1 \mathrm{~atm}$

\begin{tabular}{lllllll}
\hline Parameters & $\begin{array}{l}\text { density } \\
\left(\mathrm{kg} / \mathrm{m}^{3}\right)\end{array}$ & $\begin{array}{l}\text { thermal conductivity } \\
\lambda(\mathrm{W} / \mathrm{m} \cdot \mathrm{K})\end{array}$ & $\begin{array}{l}\text { latent heat of vaporisation } \\
\Delta H(\mathrm{~kJ} / \mathrm{kg})\end{array}$ & $\begin{array}{l}\text { surface tension } \\
\sigma(\mathrm{mN} / \mathrm{m})\end{array}$ & $\begin{array}{l}\text { diffusion concentration } \\
D\left(\mathrm{~mm}^{2} / \mathrm{s}\right)\end{array}$ & $\begin{array}{l}\text { concentration } \\
c\left(\mathrm{~kg} / \mathrm{m}^{3}\right)\end{array}$ \\
\hline water & 998 & 0.6123 & 2538 & 72.69 & 24.46 & 0.017 \\
ethanol & 789 & 0.14 & 846 & 22.1 & 11.34 & 0.694 \\
\hline
\end{tabular}


Table 2 Summary of substrates physical properties

\begin{tabular}{llll}
\hline Parameters & Units & $\mathrm{Al}$ & PTFE \\
\hline Density & $\mathrm{kg} / \mathrm{m}^{3}$ & 2700 & 2200 \\
Thickness & $\mu \mathrm{m}$ & 300 & 200 \\
Roughness & $\mu \mathrm{m}$ & 5 & 0.5 \\
Specific heat capacity & $\mathrm{J} / \mathrm{kg} \cdot \mathrm{K}$ & 913 & 1050 \\
Thermal conductivity & $\mathrm{W} / \mathrm{m} \cdot \mathrm{K}$ & 237 & 0.25 \\
\hline
\end{tabular}

also needed to regard temperature influence. Therefore, the basic model of evaporation rate was generalized by:

$-\frac{d m}{d t}=\pi D\left(T_{s}\right) R\left(\left(c_{s}\right)-c(\infty)\right) f(\theta)$

Moreover, it is important to note that another parameter evaporation flux also could describe the rate of mass loss, which means the evaporation rate per unit area. For drops that remain axisymmetric, the evaporation flux $j(t)$ could be calculated, using contact area $A(t)=\pi R(t)^{2}$ and volume reduction with time $\Delta V(t)$, according to the expression:

$j(t)=\frac{\rho}{\pi R(t)^{2}} \frac{\Delta V(t)}{\Delta t}$

\section{Experimental Setup}

The experimental setup involved the sessile droplet evaporation with a controlled volume on the hot isothermal substrate. The schematic setup of the experimental apparatus was shown in Fig. 4. Relevant thermophysical properties of liquids used in present experiments were listed in Table 1. Droplet volume was dominated by the priming system with high precision controlling. Experimental cell $\left(80 \times 60 \times 60 \mathrm{~mm}^{3}\right)$ was covered by optical glass around in order to obtain the evolution of droplet shape information by the charge-coupled device (CCD) camera with cold light resource (LED). The base was an aluminum cylinder $(\phi 10 \mathrm{~mm})$ with a $0.7 \mathrm{~mm}$ hole in the middle for injecting the liquid from the bottom onto the substrate. At the top of the base, we coated two different substrates: aluminum(Al) and Teflon (PTFE), the substrates' physical property data were summarized in Table 2.

To investigate the thermal effect of different substrates, we used a thin-film heater to heat the base at the bottom and a thermocouple fitted with a PID (Proportion Integration Differentiation) temperature regulator to make sure the temperature of substrate reaching the setting value $\left(T_{S}\right)$ ranging from ambient temperature to $80{ }^{\circ} \mathrm{C}$. In order to measure the variation of heat flux density, a heat flux meter was inserted connecting with a data acquisition system (Agilent 34970A). Ambient temperature and air humidity were measured with mercurial thermometer and hygrometry.

Experiment cell was fixed on the vibration isolation platform with a small vent on the top. Thus, every experiment was carried out in the atmosphere of air, the temperature and pressure was separately kept at $T_{a}=20{ }^{\circ} \mathrm{C}\left( \pm 1{ }^{\circ} \mathrm{C}\right)$ and $P=1 \mathrm{~atm}$. All the experimental data were conveyed to the data collection and analysis system which could calculate the radius $R$, height $h$, volume $V$ and contact angle $\theta$ by the analysis software and the time-varying heat flux density value from Agilent instrument.

\section{Results and Discussion}

In our experiment, two different drops (water \& ethanol) were placed on two different substrates (Al \& PTFE) in the condition of substrate heated or non-heated. The evaporation process of liquid drops on the solid substrates involved many influences. Making use of the above mentioned experimental apparatus and measuring techniques, drop's shape and contact angle were obtained to reflect the spreading behaviors, the average evaporation rate, heat flux density, and evaporation flux were measured to show the phase transition and heat supply process of the macroscopic-scale drop.
Fig. 5 AFM picture of the $\operatorname{PTFE}($ left $)$ and $\mathrm{Al}($ right $)$ coating
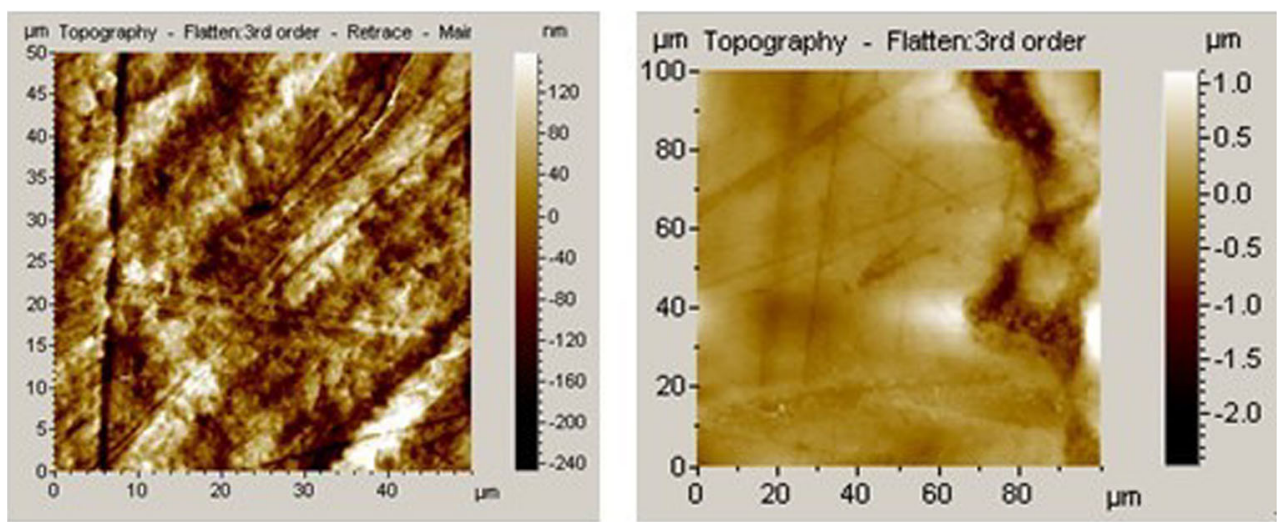


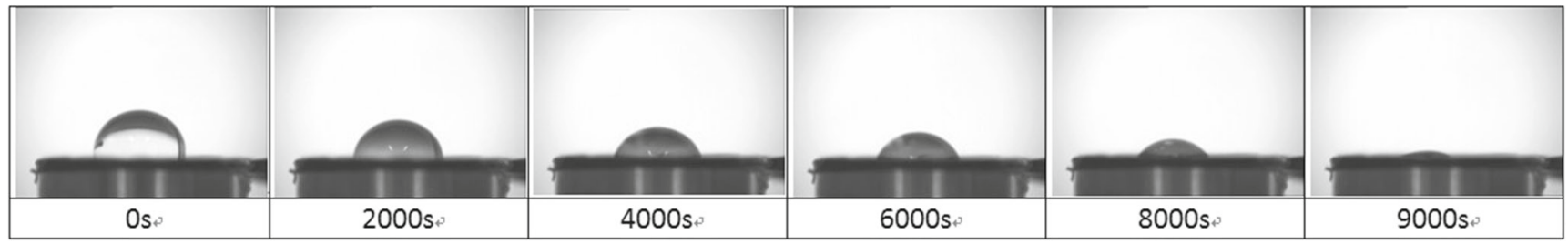

Fig. 6 Evolution of the $20 \mu \mathrm{L}$ drop shape at different time for water sessile drop on PTFE

The surface of the substrate was characterized with respect to morphology using an atomic force microscope(AFM). The local roughness was 500nm and $5 \mu \mathrm{m}$ respectively for PTFE and $\mathrm{Al}$ seen in the Fig. 5.

\section{Drop Shape Evolution}

As we know, the contour of a drop was very sensitive to gravity level. The contour of an initial volume of $20 \mu \mathrm{L}$ water drop evaporating on the PTFE at different moments were shown in Fig. 6. Compared with the experimental data at the low gravity level by Zhu et al. (2010), drop's shape on ground seemed flattened at early stage so that spherical cap shape could not correspond to the macroscopic-scale droplet. But after a few moments when the diameter reduced to the capillary length or even less than it, the contour fitted the assumption of spherical cap well. Therefore, all the expression of the prerequisite for spherical cap was not suitable for macroscopic-scale drop since it was a varying procedure.

For water droplet evaporating on Al or PTFE behaved in a similar manner, an example of the evaporation evolution of droplet base radius, height and contact angle for a droplet of initial volume $V_{0}=20 \mu \mathrm{L}( \pm 1 \mu \mathrm{L})$ in the environmental condition: $T_{a}=20{ }^{\circ} \mathrm{C}\left( \pm 1{ }^{\circ} \mathrm{C}\right), H=45 \%( \pm 2 \%)$ was presented in Fig. 7.

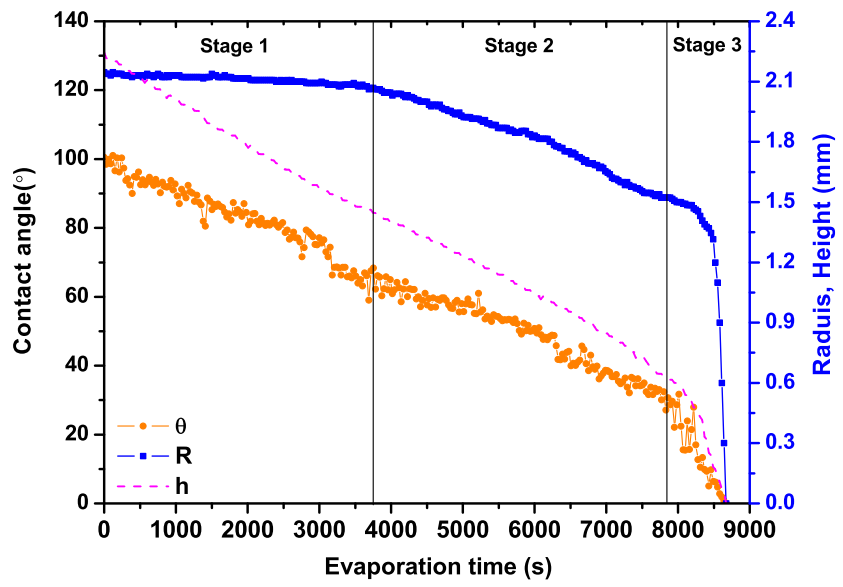

Fig. 7 Evolutions of droplet base radius, height and contact angle for a water drop
It could be seen in the picture that macroscopicscale droplet evaporation had three stages which in good agreement with the result of most previous literatures like Bourges-Monnier and Shanahan (1995). First stage was constant contact area, the phase change process of evaporation drop decreased the contact angle, and the contact line remained constant due to the adhesion force induced by roughness substrate. When the contact angle reduced to a critical value, the horizontal components of the surface tensions could not balance the adhesion force, then the triple line started to depin, this was the second depin stage. Compared with the small volume drop, the contact angle of the macroscopic-scale droplet did not kept constant, but descended with a low rate. And the final flushing stage presented the fast decreasing of base radius, height and contact angle which implied an overall drying tendency.

\section{Evaporation Rate}

Average evaporation rate was an important parameter representing the rate of mass loss from a drop. It equals to the ratio of the initial mass or volume to the total evaporation time. To complete understand the mechanism of heat and mass transfer, various drop sizes ranging from $10 \mu \mathrm{L}$ to $40 \mu \mathrm{L}$ were done. Our experimental results plotted in Fig. 8 were denoted as solid dots. Moreover, Dunn et al. (2009) results of which drop volume ranging from 0.5 to $8 \mu \mathrm{L}$ not to exceed the capillary length were also drawn with hollow dots, the solid line stands for curve fitting. Noting that the environmental condition was $T_{a}=22{ }^{\circ} \mathrm{C}, P=99.8 \mathrm{kPa}$, $H=40 \%( \pm 2 \%)$ for them and $T_{a}=20{ }^{\circ} \mathrm{C}\left( \pm 1{ }^{\circ} \mathrm{C}\right)$, $P=1 \mathrm{~atm}, H=47 \%( \pm 2 \%)$ for us.

Figure 8 displayed that the average evaporation rate was approximately a linear function of the droplet diameter. The results also could be explained by the Eq. 4. It was obvious that whether the drop was large or small, water liquid evaporating on $\mathrm{Al}$ had higher rates than on PTFE. The reason is that $\mathrm{Al}$ has higher thermal conductivity than PTFE, therefore evaporative cooling on an Al substrate was much less so that water with the same volume evaporates faster. By contrast, the surface area of our experiments was larger than what Dunn did, so that the evaporation rate was higher 


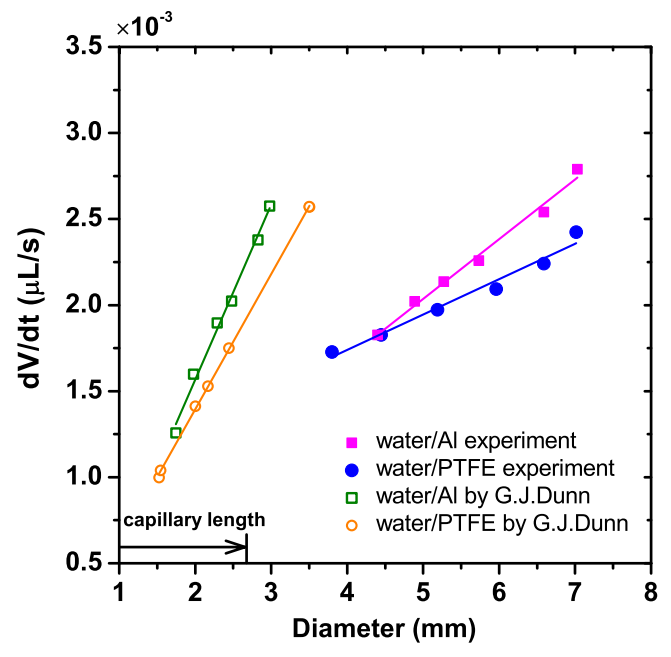

Fig. 8 Average evaporation rate as a function of droplet radius for various water drop sizes deposited on Al and PTFE substrate. Present results are compared with Dunn et al. (2009)

for accelerating the vapor concentration diffusing into the air.

In order to get more insight into the thermal effect of substrates for macroscopic-scale drop, we proposed different water drop on $\mathrm{Al}$ substrate heating from 20 to $80{ }^{\circ} \mathrm{C}$. The average evaporation rate was then plotted versus the temperature difference $\left(\Delta T=T_{s}-T_{a}\right)$ of $\mathrm{Al}$ substrate in Fig. 9 (triangle dots represented the experimental results of $20 \mu \mathrm{L}$ water, circle dots represented the experimental results of $40 \mu \mathrm{L}$ water, and dashed line represented the basic model value).

According to previous reference (Ghasemi and Ward 2011), energy required to evaporate a liquid was mainly consisted of thermal conduction, thermocapillary convection and buoyant convection. As shown in Fig. 9, it was

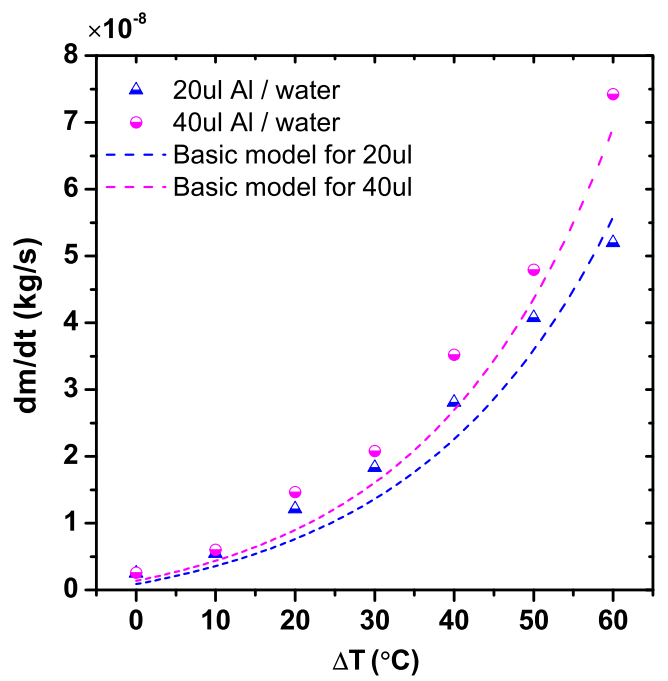

Fig. 9 Average evaporation rate versus the temperature difference of Al substrate $\Delta T$ for different volume evidenced that with the temperature difference becomes larger, the effects of thermocapillary and buoyant convection strengthened and the evaporation rate was rapidly growth. It's worth noticing that for large size droplet, the distinction would be expanded as the substrate's temperature was higher and higher. Since increasing the substrate's temperature amplified the buoyant convection effect, then the inside flow would be accelerated so that the evaporation mass rate was higher. In addition, by contrast to the basic model, it had a good agreement with the tendency of the experimental data, but the difference illustrated that diffusion model neglected the effect of internal flow so that the value was lower. We could make a conclusion that with the temperature difference increasing, droplet evaporation affected by the comprehensive influence of heat conduction, thermocapillary convection and buoyant convection.

\section{Heat and Mass Transfer}

Consequently, we focused on heat and mass transfer at the liquid-vapor interface. At beginning, heat flux density evolution was discussed to study the energy absorption by the drop from the hot substrate. Second, we analysed mass distribution by presenting the evaporation mass flux during the whole process.

With the drop evaporation, it was necessary to analyze the heat flux density variation over time. We measured working liquids at the same initial volume of $20 \mu \mathrm{L}$ evaporating on different substrates in Fig. 10. It confirmed that at the beginning of the heating process, probably $10 \%$ of the droplet lifetime, the cold fluid warmed soon. This also could be described as an initial transient period: droplet absorbed energy from the heated substrate, as a result, heat flux density gone up dramatically. Then a quasi-steady state during most of the lifetime could be seen. Finally, heat flux density

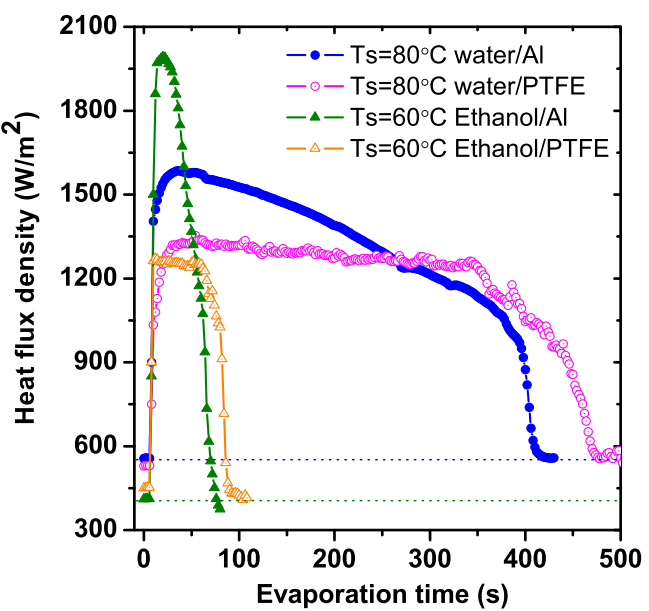

Fig. 10 Heat flux versus evaporation time of different liquids on different heated substrates, $V_{0}=20 \mu \mathrm{L}$ 


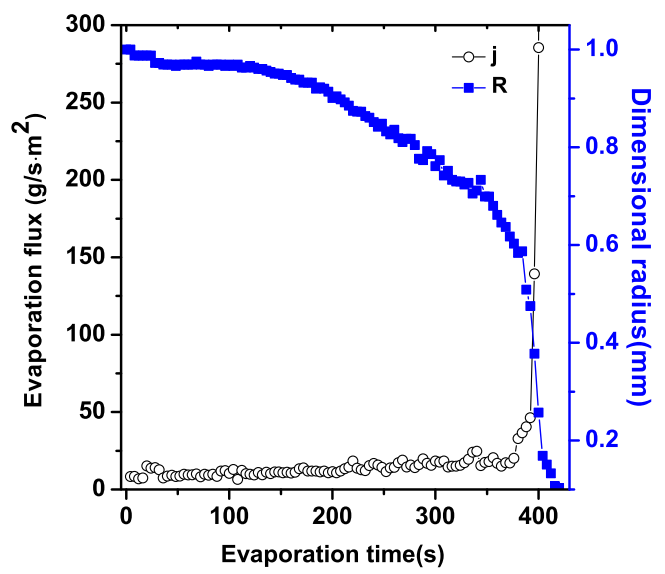

Fig. 11 Evolution of water droplet evaporation flux with time on $\mathrm{Al}$ $\left(T_{s}=80^{\circ} \mathrm{C}\right)$

dropped rapidly to the initial value which means the evaporation process completely finish. It could be noticed that no matter what liquid it was, the heat flux density seemed stable as time goes by when evaporating on the PTFE. The insulating substrate had poor thermal conductivity must be the reason.

In terms of evaporation flux $j$ which means the evaporation mass transfer at unit area as it mentioned in expression (7), Fig. 11 exhibited the evolution of a $20 \mu \mathrm{L}$ water droplet evaporating on $\mathrm{Al}$ substrate at $T_{s}=80^{\circ} \mathrm{C}$. The dimensional radius means the contact radius at each moment versus the initial radius, which showed the pinned mode(keep stable) and de-pinned mode(decrease with time). It could be seen that the mass flux was slowly increasing during quasi-steady state owing to the surface area decreasing and volume variation over time was linear. While it suddenly enlarged at the quick-drying stage on account of the surface area that shrinked rapidly at the flushing period. The tendency fully coincided with the numerical analysis by Saada and Tadrist (2012).

\section{Conclusion}

In summary, experiments of sessile macroscopic-scale drops evaporating on heated substrates have been investigated. The drop's shape variation(volume, contact angle, contact radius and height), average evaporation rate, heat flux density, and evaporation flux were taken into account to study the evaporation rules for macroscopic-scale droplet.

It is found that lifetime of evaporating water droplet could be separated into three stages, first, constant contact area, the phase transition decreased the contact angle keeping the contact line unchangeable, then the triple line started to depin with decreasing of contact angle, ended up with the flushing stage. Although the macroscopic-scale droplet affected by the gravity effect and the assumption of the spherical cap shape was not suitable, the average evaporation rate still exhibited a linear relation with the contact diameter as well as the small drops. For the thermal effect of different substrates, results revealed that the evaporation rate was higher for substrates with higher thermal conductivity like aluminum. And with the increasing of temperature difference, especially for larger volume drops, thermocapillary convection and buoyant convection strengthened corresponding to the increasing of evaporation rate. It was necessary to analyze the evaporation rate per unit area-evaporation flux, slowly increased during quasisteady state, but enlarged dramatically at the flushing stage. The mechanism of energy transport during evaporation process was also studied. According to the experimental results of heat flux density, it seemed that droplet absorbed energy from the heated substrate, then transported to the liquidvapor interface providing the energy for the evaporation. Furthermore, we are looking forward to a more accurate model which could be used to predict the whole evaporation process.

Acknowledgments This research was financially supported by the National Natural Science Foundation of China (Grants No.11302236) and the Strategic Priority Research Program on Space Science, Chinese Academy of Sciences (Grants No.XDA 04073000, XDA 04020202-02), and China Manned Space Program (TZ-1).

Conflict of interests The authors declare that there is no conflict of interest.

\section{References}

Bourges-Monnier, C., Shanahan, M.E.R.: Influence of evaporation on contact angle. Langmuir 11, 2820-2829 (1995)

Deegan, R.D., Bakajin, O., Dupont, T.F., Huber, G., Nagel, R.S.: Contact line deposits in an evaporating drop. Phys. Rev. E. 62, $756 \mathrm{C} 765$ (2000)

Dittrich, P.S., Manz, A.: Lab-on-a-chip: microfluidics in drug discovery. Nat. Rev. Drug Discov. 5, 210-218 (2006)

Dunn, G., Wilson, S.K., Duffy, B.R., David, S., Sefiane, K.: The strong influence of substrate conductivity on droplet evaporation. J. Fluid Mech 623, 329-351 (2009)

Erbil, H.Y.: Evaporation of pure liquid sessile and spherical suspended drops: A review. Adv. Colloid Interface Sci. 170, 67-86 (2012)

Ghasemi, H., Ward, C.A.: Mechanism of sessile water droplet evaporation: Kapitza resistance at the solid-liquid interface. J. Phys. Chem. C. 115, 21311-21319 (2011)

Gogos, G., Sadhal, S.S.: Thin-flame theory for the combustion of a moving liquid drop: Effects due to variable density. J. Fluid Mech. 17, 121-144 (1986)

Hu, H., Larson, R.G.: Evaporation of a sessile droplet on a substrate. J. Phys. Chem. B. 106, 1334C1344 (2002)

$\mathrm{Hu}, \mathrm{H}$., Larson, R.G.: Analysis of the effect of marangoni stresses on the microflow in an evaporating sessile drop. Langmuir 21, 3972 3980 (2005) 
Jing, J., Reed, J.: Automated high resolution optical mapping using arrayed, fluid-fixed DNA molecules. Proc. Natl. Acad. Sci. 95, 8046-8051 (1998)

Picknett, R.G., Bexon, R.: The evaporation of sessile or pendant drops in still air. J. Colloid Interface Sci. 61, 336 (1977)

Ruiz, O.E., Black, W.Z.: Evaporation of water droplets placed on a heated horizontal surface. J. Heat Transfer 124, 854-863 (2002)

Saada, M.A., Tadrist, L.: Evaporation of a sessile drop with pinned or receding contact line on a substrate with different thermophysical properties. Int. Heat Mass Transfer 58, 197 (2013)

Saada, M.A., Tadrist, L.: Effect of substrate thickness and thermal conductivity on an evaporating sessile drop. J. Phys. Conf. Series 395, 012140 (2012)
Sobac, B., Brutin, D.: Thermal effects of the substrate on water droplet evaporation. Phys. Rev. E 86, 021602 (2012)

Tadmor, R., Bahadur, P.: Measurement of lateral adhesion forces at the interface between a liquid drop and a substrate. Phys. Rev. Lett 103, 266101 (2009)

Young, T.: An essay on the cohesion of fluids. Philos. Trans. Royal Soc. Lond. 95, 65-87 (1805)

Zhu, Z.Q., Brutin, D., Liu, Q.S., Wang, Y., Mourembles, A., Xie, J.C., Tadrist, L.: Experimental investigation of pendant and sessile drops in microgravity. Microgravity Sci. Technol 22, 339-345 (2010)

Zhu, Z.Q., Wang, Y., Liu, Q.S., Xie, J.C.: Influence of bond number on behaviors of liquid drops deposited onto solid substrates. Microgravity Sci. Technol. 24, 181-188 (2012) 